\section{Psoriasis, biological drugs and Coronavirus Disease 2019: Real life experience of two Italian provinces}

\author{
Martina Vispi, ${ }^{1}$ Teresa Corradin, ${ }^{2}$ \\ Camilla Peccianti, ${ }^{1}$ Luca Feci, ${ }^{1}$ \\ Laura Casini, ${ }^{1}$ Chiara Pisani, ${ }^{1}$ \\ Susanna Fabbroni, ${ }^{1}$ Paola Corsetti, ${ }^{2}$ \\ Margherita Croatto, ${ }^{2}$ \\ Michele Pellegrino ${ }^{1}$ \\ ${ }^{1}$ Dermatology Unit, Misericordia \\ Hospital, Grosseto; '2Dermatology Unit, \\ Pordenone Hospital, Pordenone, Italy
}

\begin{abstract}
On January 30, 2020, World Health Organization (WHO) stated that a new coronavirus disease outbreak [COronaVIrus Disease - 19 (COVID-19)] was an international public health emergency.

Many news, often fake ones, about the derived pandemic rapidly spread along the media, thus leading many dermatological patients to identify as "risk category" and sometimes discontinue treatments by themselves. The Dermatology Units in Grosseto and Pordenone simultaneously carried out a data collection by remote-conducted visits, evaluating the incidence of COVID-19 in psoriatic patients. Only 1 patient (close contact of a case) as part of the psoriasis analyzed group was tested and turned out to be positive for SARS-CoV-2, developing no symptoms during the observation period. The collected information may suggest that psoriasis, biotechnologically treated or not, cannot promote or aggravate the clinical trend of the SARS-CoV-2 infection, hence stopping systemic therapy in negative or clinically free SARS-CoV-2 patients is not recommended in general.
\end{abstract}

\section{Introduction}

On December 31, 2019, Chinese health authorities reported that an epidemic outbreak of cases of atypical pneumonia of unknown etiology had developed in the city of Wuhan (central-eastern China). A few days later, the Chinese Center for Disease Control announced that the causative agent, a new coronavirus provisionally called 2019 - new CoronaVirus (2019-nCoV), had been identified. ${ }^{1}$

On 30 January 2020, World Health Organization (WHO) stated that the new coronavirus outbreak, then called Severe
Acute Respiratory Syndrome - Coronavirus - 2 (SARS-CoV-2), responsible of the so called COronaVIrus Disease - 19 (COVID19), was an international public health emergency. ${ }^{2}$

After its sequencing, the International Committee on Taxonomy of Viruses (ICTV) developed its taxonomy, ${ }^{3}$ confirming that it is a virus of the Coronaviridae family, which shares $79.5 \%$ of the gene sequence with the Severe Acute Respiratory Syndrome (SARS) coronavirus ${ }^{4}$ and $96.2 \%$ with that of a bat coronavirus (Rhinolophus affinis species) which is supposed to be the animal reservoir species with direct transmission to humans or through other intermediate hosts, not better identified yet. ${ }^{5}$ WHO reiterated that possible interhuman transmission modalities were by air, with saliva and aerosol from secretions of the upper airways, through conjunctival and oral mucous membranes, by direct contact and/or by oral-fecal route. Although the main transmission mechanism is linked to contact with people who have contracted the infection and are symptomatic for the disease, the possibility of transmission from infected but asymptomatic people has not been denied, ${ }^{6}$ similarly to what is already known for other coronaviruses like the Middle East Respiratory Syndrome Coronavirus (MERS-CoV).

The SARS-CoV-2 pandemic, as a "health tsunami", quickly conquered the front pages of newspapers, news programs, websites. Data and information quickly and frantically followed each other and often became a source of fake news and baseless scientific information. In particular, social media have given communication opportunities to anyone with access to the network with the inevitable consequence that the message transmitted often turns out to be distorted, de-contextualized or even untrue. The consequence of this redundancy of information ${ }^{7}$ often of inadequate and confusing quality has led many patients with chronic dermatological pathologies such as psoriasis, Hidradenitis Suppurativa (HS), or atopy to identify themselves as "risk category" not only for the underlying pathology, but also and above all for the biotechnological drugs they were in therapy with, suspending them on their own initiative. This selfmanagement probably took place to protect themselves from what many patients call the "magnet effect", almost as if the dermatosis and/or the "immunosuppressant" drug were capable of magnetic attraction towards SARS-CoV-2 and so COVID-19.

Trying to stem this invasive phenomenon and to evaluate at the same time the incidence of COVID-19 in dermatological patients, focusing on psoriatic ones, the
Correspondence: Martina Vispi, Dermatology Unit, Misericordia Hospital, Via Senese 161 58100 Grosseto, Italy

Tel.: +390564 485386

E-mail: martinavispi@yahoo.it

Key words: COVID-19; Psoriasis; Biological Drugs.

Conflict of Interests: The Authors declare no potential conflict of interests.

Received for publication: 22 May 2020

Accepted for publication: 11 June 2020

This work is licensed under a Creative Commons Attribution-NonCommercial 4.0 International License (CC BY-NC 4.0).

( Copyright: the Author(s), 2020

Licensee PAGEPress, Italy

Dermatology Reports 2020; 12:8642

doi:10.4081/dr.2020.8642

Dermatology Units in Grosseto and Pordenone simultaneously carried out a data collection, by remote-conducted visits, concerning hundreds of psoriasis patients being treated with biological drugs.

\section{Materials and Methods}

For this purpose, 246 patients (104 females and 142 males), in an age range of 21-90 and with a mean age of 56 years old (Figure 1), with moderate-severe psoriasis receiving biologic or small molecular drug therapy on March 1, 2020, underwent a virtual check-up and a series of pandemic-related questions during the two last weeks of April. All patients were contacted and interviewed through the aid of different video/audio devices (smartphone, computer, tablet or phone), according to what the individual patient possessed; they were divided into different groups based on the biotechnological or small molecular drug in use (Table 1).

Regardless of the ongoing therapy, only 1 patient, in therapy with ustekinumab (Stelara, Janssen Biotech Inc, Horsham, PA) tested positive for SARS-CoV-2, although asymptomatic. The swab, which had allowed the identification of the subject and the consequent home isolation, had been carried out because of the positivity of a cohabitant who had complained of persistent fever and difficulty in breathing. This patient, who had been carrying out therapy with the biotech drug on a quarterly basis, as per therapeutic protocol, was formally declared SARS-CoV-2 free after perfor- 
ming two subsequent negative swabs.

Neither swabs nor serological surveys were performed in the residual group of patients.

10 patients [in therapy with ustekinumab (3 of them), secukinumab (3), apremilast (2), certolizumab and ixekizumab (1 each)] complained of respiratory and/or gastrointestinal disorders, but they didn't undergo swab or serology as fever or further suspicious criteria were absent.

8 asymptomatic patients [taking secukinumab, etanercept or adalimumab (2 each) and ustekinumab or ixekizumab (1 each)] voluntarily stopped their own therapy, fearing that drug-induced immunosuppression would facilitate the infection regardless of compliance with behavioural standards. Although flu vaccination was recommended to all patients, in the last quarter of
2019 only 36 patients over the age of 50 underwent it.

\section{Results}

In the provinces of Grosseto and Pordenone, as of May 12, 2020, 421 and 654 COVID-19 positive subjects were respectively registered (totalling 1075 cases), compared to a resident population of 221,629 and 312,794 inhabitants each, for a total of 534,423 residents [Istituto Nazionale di Statistica (ISTAT) 2019 data].

The percentage of COVID-19 positivity recorded across the entire population residing in the 2 provinces was lower $(0.20 \%)$ than the national one (around $0.33 \%$ ), even though the latter is burdened by a wide

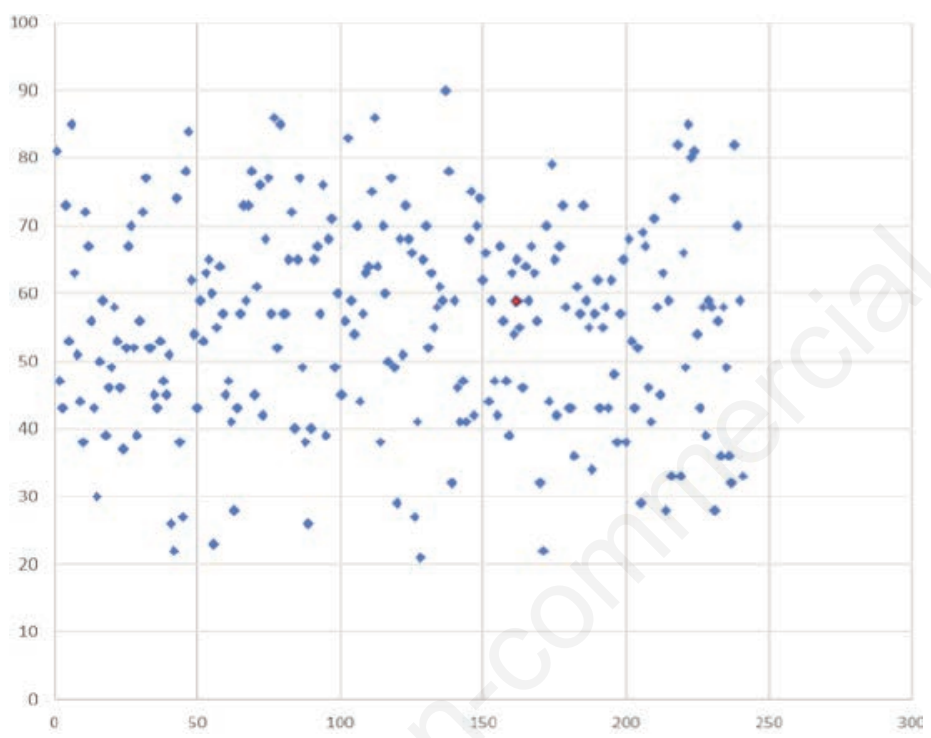

Figure 1. Case distribution by age (age range 21-90 years old; mean age 56 years old). SARS-CoV-2 positive cases are shown in red.

Table 1. The patients were divided into different groups based on the biotechnological or small molecular drug in use.

\begin{tabular}{lcc} 
Ongoing therapy & Number of patients & Percentage of patients \\
Ustekinumab & 61 & $25 \%$ \\
Etanercept & 55 & $22 \%$ \\
\hline Secukinumab & 37 & $15 \%$ \\
Adalimumab & 36 & $15 \%$ \\
\hline Apremilast & 22 & $9 \%$ \\
Ixekizumab & 14 & $6 \%$ \\
\hline Guselkumab & 10 & $4 \%$ \\
Risankizumab & 6 & $3 \%$ \\
\hline Infliximab & 3 & $1 \%$ \\
Brodalumab & 1 & $0 \%$ \\
\hline Certolizumab & 1 & $0 \%$ \\
\hline
\end{tabular}

variability from area to area.

Only 1 patient (close contact of a case) as part of the psoriasis analyzed group was tested and turned out to be positive for SARS-CoV-2, developing no symptoms during the observation period. The collected information may suggest that psoriasis, biotechnologically treated or not, cannot promote or aggravate the clinical trend of the SARS-CoV-2 infection, hence stopping systemic therapy in negative or clinically free patients is not recommended in general.

\section{Discussion}

Despite what has been circulating by some news outlets, our data are in line with what has been recorded by the Higher Institute of Health and document that psoriasis, regardless of the use of biologic drugs, is not a chronic comorbidity which can promote or aggravate the clinical trend of the SARS-CoV-2 infection. With regard to biotechnology therapies, it is likely that these drugs, by significantly reducing the inflammatory cascade typical of psoriasis, may even help to reduce the cytochinic storm recorded in many positive COVID-19 patients.

Lebwohl et al. have recently published in the literature overall infection rates as well as rates of upper respiratory tract infections and nasopharyngitis for each available biologic agent versus its placebo control by extrapulating published data from pivotal trials ${ }^{8}$. They suggest that, even if it is not known wheter biologic therapies make patients being more susceptible to coronavirus, in the pre-coronavirus era, respiratory infection percentages were similar to those with placebo. Discontinuation of some biologics could have worse effects, such as loss of response when treatments are reintroduced or even result in the formation of antibodies to the discontinued biologic. As therapy prosecution during COVID19 emergency seems to strictly depend on the quality of information that patients acquire, ${ }^{9}$ dermatologists should inform them correctly and exhaustively to increase patients' adherence to treatments.

In another paper written by Brownstone et al., similar considerations are made; ${ }^{10}$ however, it is pointed out that, as SARSCoV-2 is a novel pathogen associated with mortality in a subset of patients, a cautious approach is warranted and so that treatment decisions should be made taking into consideration each patient's individualized condition. Actually, treatment decisions should always be made on the basis of dialogue between patient and physician; fortunately, 
all this could take place during the lockdown period too, by remote surveillance of patients. An italian experience in teledermatologic monitoring showed that it was effective in preventing unnecessary worsening of severe chronic skin diseases and poor outcomes due to withdrawal of current therapy. ${ }^{11}$

One thing to take into consideration too, is the lack of adherence to vaccination programmes (around 15\%), probably to be linked to the media wave of "no-Vax", which could open up negative scenarios once a useful vaccine against SARS-CoV-2 is obtained.

Both our real life data and current evidence recommend not to stop systemic psoriasis therapy in negative or clinically free SARS-CoV-2 patients. Our experience aims to be a small contribution to spreading the message of not interrupting current therapy and keeping in touch with one's own dermatologist for any clarification and doubt.

\section{References}

1. Qun Li, Xuhua Guan, Peng Wu, et al. Early transmission dynamics in Wuhan, China, of novel coronavirus-infected pneumonia. New Engl J Med 2020;382: 1199-207.

2. WHO. Statement on the second meeting of the International Health Regulations (2005) Emergency Committee regarding the outbreak of novel coronavirus (2019-nCoV). World Health Organization, 2020. Available from: https://www.who.int/news-room/ detail/30-01-2020-statement-on-thesecond-meeting-of-the-internationalhealth-regulations-(2005)-emergencycommittee-regarding-the-outbreak-ofnovel-coronavirus-(2019-ncov).

3. Coronaviridae Study Group of the International Committee on Taxonomy of Viruses The species Severe acute respiratory syndrome-related coronavirus: classifying 2019-nCoV and naming it SARS-CoV-2 Nat Microbiol 2020;5: 536-44.

4. Awadasseid $\mathrm{A}, \mathrm{Wu} \mathrm{Y}$, Tanaka $\mathrm{Y}$ and Zhang W Initial success in the identification and management of the coronavirus disease 2019 (COVID-19) indicates human-to-human transmission in Wuhan, China Int J Biol Sci. 2020; 16:1846-60.

5. Decaro N, Lorusso A Novel human coronavirus (SARS-CoV-2): a lesson from animal coronaviruses Vet Microbiol. 2020;244:108693.

6. WHO. Q\&A on coronaviruses (COVID-19). World Health Organization, 2020. Available from: https://www.who.int/emergencies/disea ses/novel-coronavirus-2019/questionand-answers-hub/q-a-detail/q-a-coronaviruses.

7 Lambert TW, Soskolne CL, Bergum V et al. Ethical perspectives for public and environmental health: fostering autonomy and the right to know. Environ Health Perspect 2003;111:133-7.

8 Lebwohl M, Rivera-Oyola R, Murrel DF. Should biologics for psoriasis be interrupted in the era of COVID-19? J Am Acad Dermatol 2020;82:1217-18. doi: 10.1016/j.jaad.2020.03.031.

9. Bragazzi NL, Riccò M, Pacifico A, Malagoli P, Kridin K, Pigatto P, Damiani G. COVID-19 knowledge prevents biologics discontinuation: data from an Italian multi-center survey during RED-ZONE declaration. Dermatol Ther 2020;e13508. doi: 10.1111/dth. 13508 .

10. Brownstone ND, Thibodeaux QG, Reddy VD, et al. Novel coronavirus disease (COVID-19) and biologic therapy in psoriasis: infection risk and patient counseling in uncertain times. Dermatol Ther 2020;10:339-49.

11. Brunasso AMG, Massone C. Teledermatologic monitoring for chronic cutaneous autoimmune diseases with smartworking during Covid-19 emergency in a tertiary center in Italy. Dermatol Ther 2020;e13495. 